This article was downloaded by: [Harvard University]

On: 15 August 2008

Access details: Access Details: [subscription number 788777878]

Publisher Taylor \& Francis

Informa Ltd Registered in England and Wales Registered Number: 1072954 Registered office: Mortimer House, 37-41 Mortimer Street, London W1T 3JH, UK

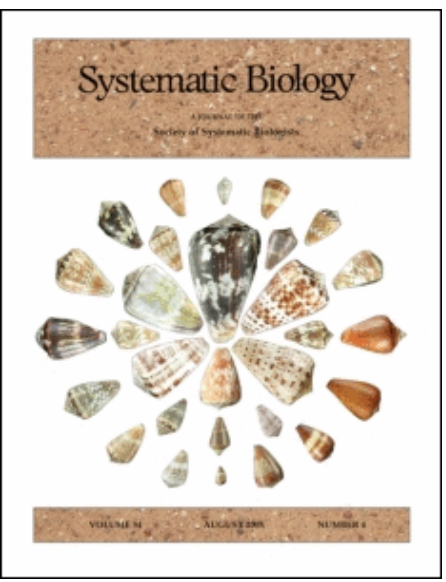

\title{
Systematic Biology
}

Publication details, including instructions for authors and subscription information:

http://www.informaworld.com/smpp/title content=t713658732

\section{Phylogenetic Signal, Evolutionary Process, and Rate}

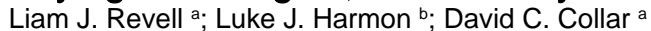

a Department of Organismic and Evolutionary Biology, Harvard University, Cambridge, Massachusetts, USA ${ }^{\mathrm{b}}$

Department of Biological Sciences, University of Idaho, Moscow, Idaho, USA

First Published on: 01 August 2008

To cite this Article Revell, Liam J., Harmon, Luke J. and Collar, David C.(2008)'Phylogenetic Signal, Evolutionary Process, and Rate',Systematic Biology, 57:4,591 - 601

To link to this Article: DOI: $10.1080 / 10635150802302427$

URL: http://dx.doi.org/10.1080/10635150802302427

\section{PLEASE SCROLL DOWN FOR ARTICLE}

\footnotetext{
Full terms and conditions of use: http://www.informaworld.com/terms-and-conditions-of-access.pdf

This article may be used for research, teaching and private study purposes. Any substantial or systematic reproduction, re-distribution, re-selling, loan or sub-licensing, systematic supply or distribution in any form to anyone is expressly forbidden.

The publisher does not give any warranty express or implied or make any representation that the contents will be complete or accurate or up to date. The accuracy of any instructions, formulae and drug doses should be independently verified with primary sources. The publisher shall not be liable for any loss, actions, claims, proceedings, demand or costs or damages whatsoever or howsoever caused arising directly or indirectly in connection with or arising out of the use of this material.
} 


\title{
Phylogenetic Signal, Evolutionary Process, and Rate
}

\author{
LiAM J. REVELL, ${ }^{1}$ LUKe J. HARMON, ${ }^{2}$ AND DAVID C. COLlAR ${ }^{1}$ \\ ${ }^{1}$ Department of Organismic and Evolutionary Biology, Harvard University, Cambridge, Massachusetts 02138, USA; \\ E-mail: lrevell@fas.harvard.edu (L.J.R.) \\ ${ }^{2}$ Department of Biological Sciences, University of Idaho, Moscow, Idaho 83844, USA
}

\begin{abstract}
A recent advance in the phylogenetic comparative analysis of continuous traits has been explicit, model-based measurement of "phylogenetic signal" in data sets composed of observations collected from species related by a phylogenetic tree. Phylogenetic signal is a measure of the statistical dependence among species' trait values due to their phylogenetic relationships. Although phylogenetic signal is a measure of pattern (statistical dependence), there has nonetheless been a widespread propensity in the literature to attribute this pattern to aspects of the evolutionary process or rate. This may be due, in part, to the perception that high evolutionary rate necessarily results in low phylogenetic signal; and, conversely, that low evolutionary rate or stabilizing selection results in high phylogenetic signal (due to the resulting high resemblance between related species). In this study, we use individual-based numerical simulations on stochastic phylogenetic trees to clarify the relationship between phylogenetic signal, rate, and evolutionary process. Under the simplest model for quantitative trait evolution, homogeneous rate genetic drift, there is no relation between evolutionary rate and phylogenetic signal. For other circumstances, such as functional constraint, fluctuating selection, niche conservatism, and evolutionary heterogeneity, the relationship between process, rate, and phylogenetic signal is complex. For these reasons, we recommend against interpretations of evolutionary process or rate based on estimates of phylogenetic signal. [Comparative method; evolutionary lability; functional constraint; genetic drift; niche conservatism; quantitative characters; phylogenetics.]
\end{abstract}

Observations from species related by a phylogenetic tree are often statistically nonindependent due to common ancestry (Felsenstein, 1985; Harvey and Pagel, 1991). This is because shared history leads to phenotypic similarity between related species under many evolutionary processes (Hansen and Martins, 1996). This phylogenetic dependence in the data can be accounted for using any of a variety of special statistical methods developed expressly for phylogenetic data (e.g., Cheverud et al., 1985; Felsenstein, 1985; Grafen, 1989; Garland et al., 1992; Hansen and Martins, 1996, 1997; Pagel, 1999; Rohlf, 2001).

Several recent studies (e.g., Freckleton et al., 2002; Blomberg et al., 2003) have recommended that the phylogenetic dependence of observations related by a phylogeny should not be assumed a priori but rather estimated from the tree and data. This is because under some evolutionary processes, the relationship between phenotypic similarity among species and their phylogenetic relatedness is expected to be weak (e.g., Hansen and Martins, 1996).

Phylogenetic signal, defined herein as the statistical nonindependence among species trait values due to their phylogenetic relatedness, has been estimated in numerous empirical studies. Although the measurement of a pattern (statistical dependence), phylogenetic signal is often interpreted as providing information about the evolutionary process or rate. For example, it is common to equate low phylogenetic signal with evolutionary lability (e.g., Gittleman et al., 1996; Blomberg et al., 2003; Rheindt et al., 2004; Silvertown et al., 2006; Bozinovic et al., 2007). Similarly, strong phylogenetic signal has been interpreted as a sign of niche or evolutionary conservatism (e.g., Zanne et al., 2005; Ossi and Kamilar, 2006; Swenson et al., 2007). Although the amount of phylogenetic signal in a given data set is related to the evolutionary process, currently used terms are vague. For example, "niche conservatism" or "evolutionary conser- vatism" could imply either strong stabilizing selection or a low rate of evolutionary change. By contrast, "evolutionary lability," a term that has been synonymized with low phylogenetic signal by some authors (e.g., Gittleman et al., 1996; Blomberg et al., 2003), would seem to imply rapid evolutionary change but does not specify a process (e.g., genetic drift, natural selection). An important outstanding issue, to be clarified by this study, is whether such evolutionary processes can be inferred through the measurement of phylogenetic signal.

Here, we focus on a few specific models for the evolutionary process to determine how they affect the phylogenetic signal of continuous characters and our perception of evolutionary lability and conservatism. We quantify the consequences of several specific scenarios for the evolutionary process and rate on the phylogenetic signal in species data. Our intention is not to comprehensively simulate all possible evolutionary conditions, as the numerical simulations are quite computationally intensive and the number of possible scenarios very large. Rather, we explore a broad range of evolutionary circumstances to investigate the relations between phylogenetic signal, evolutionary process, and rate.

\section{The Covariances among Species Due to Phylogenetic History}

Throughout this article, we represent and interpret phylogenetic signal-the statistical dependence among observations for species related by a phylogenetic treeas a consequence of the phylogenetic covariance between species. Phylogenetic covariance is the covariance between species values for a given trait that is due to the shared history of taxa. The measurement of phylogenetic signal involves comparing the variability of species trait values to the residual trait variability when the expected covariances among observations are first incorporated (Blomberg et al., 2003). As such, the specific meaning and implication of phylogenetic covariance merits some brief attention. 
The covariance between the values for a given trait in two species is defined as the product of the trait values for the two species, each measured as deviations from the "phylogenetic mean" for the trait. The phylogenetic mean is the ancestral state at the root node of the phylogeny, and thus also the expected value for the phenotype at any tip under common models for the evolutionary process (Rohlf, 2001; Revell, 2008). If the two species share a greater proportion of common history, then, under many circumstances, they should be more similar than average, and their expected phylogenetic covariance will be relatively high. By contrast, if two species share little to no common history, then they will probably be less similar than average, and their expected phylogenetic covariance is low.

The expected phylogenetic covariance can also be thought of as the covariance between pairs of observations for a given trait in two species across many replicates of the evolutionary process. For example, Figure 1 shows Brownian motion simulation results for two such replications and an estimate of the phylogenetic covariance matrix computed from those and an additional 98 similar simulations.

Conveniently, under a Brownian motion model of trait evolution (the most common model for the evolution of continuously valued characters, to be discussed in greater detail below), the expected covariance between species values at the tips of the tree is exactly proportional to the shared history of the taxa. By extension (because the covariance of a random variable with itself is its variance), the expected variance for the trait value at a given tip is proportional to the summed branch length from the root to that tip. Thus, in Figure 1, the expected covariance between taxa $\mathrm{B}$ and $\mathrm{C}$ will be proportional to their shared history, in this case $v_{(\mathrm{B}, \mathrm{C})}$, whereas the expected variances for the taxa $\mathrm{A}, \mathrm{B}$, and $\mathrm{C}$ are proportional to the summed branch lengths from the root to each tip, or $v_{(\mathrm{A})}, v_{(\mathrm{B})}+v_{(\mathrm{B}, \mathrm{C})}$, and $v_{(\mathrm{C})}+v_{(\mathrm{B}, \mathrm{C})}$, respectively (Fig. 1). Because taxon A shares no common history with $B$ and $C$, in the context of the phylogenetic tree (Fig. 1), the expected covariance between $A$ and $B$ or $C$ is 0.0 . Results from simulation (Fig. 1) are in close agreement with these predictions. Note that the expected phylogenetic variances and covariances for and among species can only be computed in this way under an evolutionary model of constant-rate Brownian motion, in which variance is accumulated among lineages in direct proportion to the time separating them. For other circumstances, different phylogenetic covariances are expected (Hansen and Martins, 1996). In this article, we explore the process and rate dependency of the phylogenetic variances and covariances for species and the manner in which this dependency affects the calculation and interpretation of phylogenetic signal.

\section{The Measurement of Phylogenetic Signal}

There are several available measures of phylogenetic signal (Stearns, 1983; Cheverud et al., 1985; Gittleman and Kot, 1990; Abouheif, 1999; Pagel, 1999; Freckleton et al., 2002; Blomberg et al., 2003; reviewed in Blomberg a)

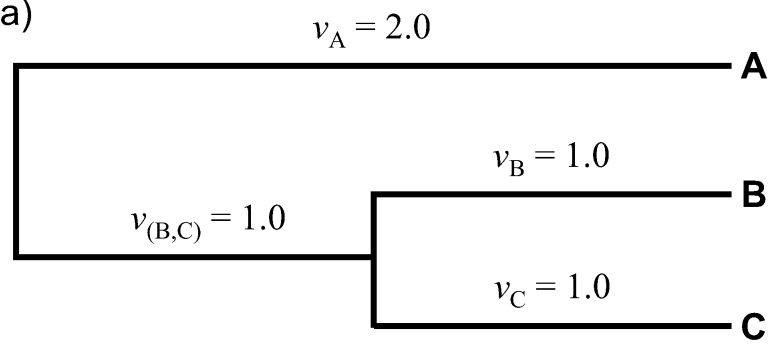

b)
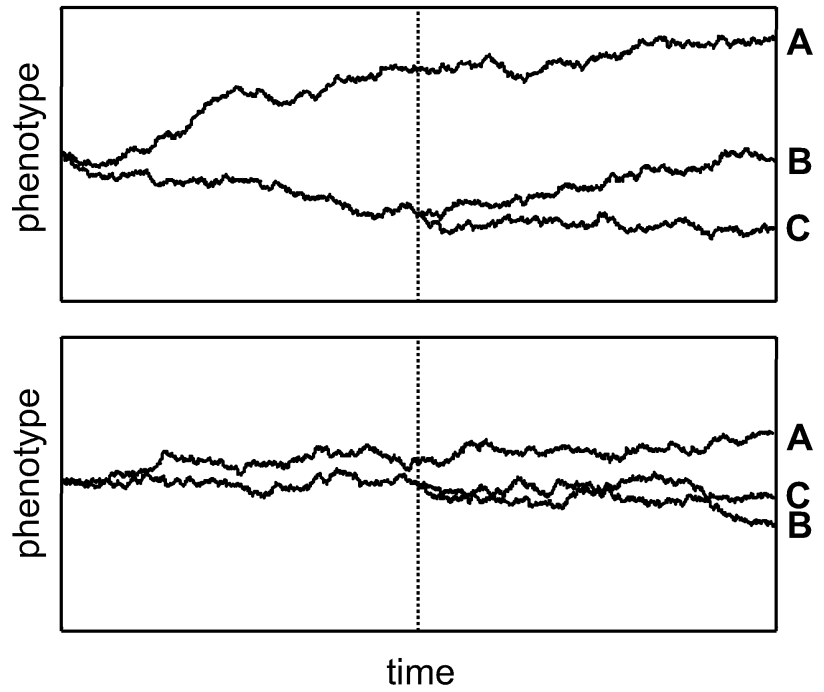

c)

Expected covariance among species $\left(\sigma^{2}=1.0\right)$ :

$$
\mathbf{C}=\left[\begin{array}{ccc}
v_{\mathrm{A}} & 0.0 & 0.0 \\
0.0 & v_{\mathrm{B}}+v_{(\mathrm{B}, \mathrm{C})} & v_{(\mathrm{B}, \mathrm{C})} \\
0.0 & v_{(\mathrm{B}, \mathrm{C})} & v_{\mathrm{C}}+v_{(\mathrm{B}, \mathrm{C})}
\end{array}\right]=\left[\begin{array}{ccc}
2.0 & 0.0 & 0.0 \\
0.0 & 2.0 & 1.0 \\
0.0 & 1.0 & 2.0
\end{array}\right]
$$

Observed covariance among species $(\mathrm{N}=100$ simulations):

$$
\overline{\mathbf{C}}=\left[\begin{array}{ccc}
1.85 & 0.11 & -0.24 \\
0.11 & 2.08 & 0.84 \\
-0.24 & 0.84 & 1.94
\end{array}\right]
$$

FIGURE 1. Covariance among species due to common history. (a) A three-taxon phylogenetic tree with branch lengths. (b) Two illustrative examples of Brownian motion evolution on the tree in (a). (c) The expected and observed phylogenetic variance-covariance matrices for the species A, B, and C in (a), in which the observed variance-covariance was obtained from the Brownian motion simulations in (b) and 98 similar simulations conducted using the phylogenetic tree in (a). The expected variance-covariance is equal, instead of merely proportional, to the matrix $\mathbf{C}$ (which consists of the times of coancestry between species) because the evolutionary rate $\left(\sigma^{2}\right)$ was set to $\sigma^{2}=1.0$ in all simulations.

and Garland, 2002; Hansen and Orzack, 2005). In addition, methods developed to compare the fit of nonphylogenetic (e.g., "pitchfork") models to phylogenetic models for the evolutionary process for continuous characters (Mooers and Schluter, 1998; Lee et al., 2006) can 
be used to obtain indirect measures of signal in evolutionary data. In this study, we focus on an evolutionary model-based metric of phylogenetic signal, $K$, proposed by Blomberg et al. (2003). $K$ is measured on the interval $(0, \infty)$, and low values indicate low phylogenetic dependence and vice versa. $K$ is computed as a function of two mean square error ratios: a mean square error ratio in which numerator and denominator are computed from the data, and the phylogenetic tree and data, respectively; and an expected mean square error ratio given the phylogenetic tree and Brownian motion as a model for the evolutionary process. Thus, $K$ has an expected value of 1.0 and otherwise provides a measure of the excess (when $>1.0$ ) or shortage (when $<1.0$ ) of statistical dependence among the tips compared to that expected when the data have evolved by Brownian motion.

Brownian motion is a commonly used model for the evolution of continuous characters (Cavalli-Sforza and Edwards, 1967; Felsenstein, 1985, 1988). It is based on the random diffusion of a particle in a fluid and is an appropriate model for the evolutionary process under genetic drift and some types of natural selection (Felsenstein, 1988, 2004; reviewed in O'Meara et al., 2006; Revell and Harmon, 2008). Under Brownian motion, the evolutionary changes along branches in the tree have an expected value of 0.0 and are normally distributed with a variance proportional to the length of the branch (Felsenstein, 1988; O'Meara et al., 2006). When evolution is by Brownian motion, variance among species accumulates in direct proportion to their time of independent evolution, which is measured by the phylogenetic branch lengths separating them (Felsenstein, 1985, 2004).

The phylogenetic signal statistic, $K$, of Blomberg et al. (2003), is computed as

$$
K=\text { observed }\left(\frac{\mathrm{MSE}_{0}}{\mathrm{MSE}}\right) / \operatorname{expected}\left(\frac{\mathrm{MSE}_{0}}{\mathrm{MSE}}\right) .
$$

In this equation, the numerator $\mathrm{MSE}_{0}$ is calculated as the mean squared error measured from the phylogenetic mean, and MSE is the mean squared error measured from the phylogenetic mean after first correcting for phylogenetic nonindependence, assuming Brownian motion. The denominator is the expected MSE ratio under Brownian motion. This equation can be equivalently expressed as follows:

$$
K=\frac{(\mathbf{y}-\hat{a} \mathbf{1})^{\prime}(\mathbf{y}-\hat{a} \mathbf{1})}{(\mathbf{y}-\hat{a} \mathbf{1})^{\prime} \mathbf{C}^{-1}(\mathbf{y}-\hat{a} \mathbf{1})} / \frac{\operatorname{tr}(\mathbf{C})-n\left(\mathbf{1}^{\prime} \mathbf{C}^{-1} \mathbf{1}\right)^{-1}}{n-1},
$$

where $\mathbf{y}$ is an $n \times 1$ vector of trait means for each of $n$ species, $\hat{a}$ is the phylogenetic mean, computed as the maximum likelihood estimate of the state at the root node of the tree, $\mathbf{1}$ is a column vector of $1.0 \mathrm{~s}, \mathbf{C}$ is an $n \times n$ matrix with elements proportional to the phylogenetic variance-covariance matrix, computed assuming Brownian motion as a model for the evolutionary process, as previously discussed (Revell and Harmon, 2008; equivalent to $\Sigma$ in Rohlf, 2001), and $t r$ indicates that the trace should be calculated. Note that only the tree (C) affects the expected MSE ratio in the denominator.

$K$ provides a measure of phylogenetic signal because if the data from the tips of the tree are hierarchically structured in a manner consistent with the phylogeny, then MSE will be small, $\mathrm{MSE}_{0}$ relatively large, and phylogenetic signal will be high (Blomberg et al., 2003). Conversely, if the structure of the data is poorly explained by the phylogenetic hypothesis, then MSE will be large, the ratio $\mathrm{MSE}_{0} / \mathrm{MSE}$ relatively small, and phylogenetic signal will be low (Blomberg et al., 2003). Note that if the tree is an equal length polytomy then the phylogenetic variance-covariance matrix, $\mathrm{C}$, is proportional to the identity matrix. In this circumstance, both numerator and denominator of the equation for $K$ will be equal to the total tree length (and thus $K$ will be 1.0) regardless of the data structure in $\mathbf{y}$.

Hypothesis tests about $K$ have been conducted using permutation (for the null hypothesis that $K$ is no greater than expected if the values for species were randomized among tips; e.g., Blomberg et al., 2003) and simulation (for the null hypothesis that $K=1.0$; e.g., Revell et al., 2007). The latter null hypothesis, that phylogenetic signal is consistent with Brownian motion, could also be tested against an analytic distribution for $K$ because, under this model, both the numerator and denominator of $K$ are expected to follow $\mathrm{F}$ distributions (S. P. Blomberg, personal communication).

We focus on $K$ rather than the similar statistic, $\lambda$ (Pagel, 1999; Freckleton et al., 2002). This is because the likelihood equation for $\lambda$ is usually not defined for values of $\lambda>1.0$ (Freckleton et al., 2002). Because $\lambda$ has an expected value of 1.0 under Brownian motion, the constraint that $\lambda$ is not defined above 1.0 limits the utility of $\lambda$ for identifying conditions that might increase phylogenetic signal over that expected under Brownian motion.

\section{METHODS}

\section{Numerical Simulations}

To explore the relationship between phylogenetic signal and evolutionary process, we conducted numerical simulations under several evolutionary conditions. These scenarios correspond to situations in which characters are evolving neutrally and under stabilizing selection, as well as situations in which parameter values are constant and in which they change through time. In our study, we conducted genetically explicit, quantitative genetic, individual-based numerical simulations on phylogenetic trees. This approach corresponds very closely to that employed in Revell (2007b) and Revell and Harmon (2008).

For our simulations we used the following quantitative genetic simulation model. We simulated diploid, hermaphroditic, sexual populations of size $N_{e}=100$. We simulated the evolution of a single quantitative trait determined by $m=20$ unlinked, additive genetic loci. We simulated mutation to a locus with an allele generation mutation rate of $\mu$ and determined the mutated 
allelic state by adding to the prior state a random effect drawn from a Gaussian distribution with variance $\alpha^{2}=0.05$, corresponding to a continuum of alleles mutation model (Crow and Kimura, 1964). We determined the value for each individual's phenotype by summing the genetic effects of all alleles at all loci. We simulated selection and reproduction by selecting, with replacement, $N_{e}$ pairs of mates with probability proportional to their fitness.

For each evolutionary scenario of interest, we simulated evolution on each of 100 pure-birth stochastic phylogenies. Each tree contained 100 taxa and was ultrametric with a total tree depth (root to tips) of 10,000 generations.

For each simulation, we first initiated the population with genetic uniformity and simulated a 1000-generation "burn-in" prior to the phylogenetic component of the simulation. We performed this burn-in to allow the ancestral population to reach mutation-selection-drift equilibrium prior to the first speciation event in the tree. We then simulated evolution for each population on each branch for the length of time specified by the branch, up to the total tree length of 10,000 generations. At each bifurcation, we duplicated the population into its daughter nodes. At the tips of each tree, we recorded the value for the phenotypic trait mean for each species in a given simulation.

Using these general conditions, we simulated eight different scenarios for the evolutionary process, which, for purposes of organizational clarity, we place in four main categories. These are as follows.

1. Genetic drift.-We simulated genetic drift using the above procedure but setting the fitness of each individual to 1.0 each generation. This corresponds to a pure drift model of evolution. To determine whether the rate of drift affected the measurement of phylogenetic signal, we simulated drift using several values for the mutation rate: $\mu=2.0 \times 10^{-5} ; \mu=2.0 \times 10^{-4} ; \mu=2.0 \times 10^{-3}$; and $\mu=2.0 \times 10^{-2}$. The rate of phenotypic evolution by genetic drift is expected to scale directly with the mutation rate according to the function:

$$
\sigma^{2}=4 m \mu \alpha^{2},
$$

in which $\sigma^{2}$ is the rate of phenotypic evolution and the remaining terms have already been defined above (Felsenstein, 1988; Falconer and MacKay, 1996; Revell and Harmon, 2008).

2. Constant selection.-We simulated two scenarios in which selection favored an optimal value or range of values for all lineages in the tree.

2A. Constant stabilizing selection.-We simulated natural selection under constant stabilizing selection on a fitness surface with a single peak using a procedure similar to that employed in Revell and Harmon (2008). We assigned individual fitness using the gaussian fitness function:

$$
w=\exp \left[-(y-\theta)^{2} /\left(2 \omega^{2}\right)\right] .
$$

In this equation, $w$ is individual fitness, $y$ is the value of the phenotypic trait, $\theta$ is the position of the phenotypic optimum, set to $\theta=0.0$ in all simulations, and $\omega^{2}$ is a parameter of the stabilizing selection surface that determines the width of the peak around the optimum. $\omega^{2}$ was set to $10,1.0 \times 10^{2}, 1.0 \times 10^{3}, 1.0 \times 10^{4}$, and 1.0 $\times 10^{5}$, which correspond to progressively weaker stabilizing selection for larger values of $\omega^{2}$ (e.g., Jones et al., 2003; Revell and Harmon, 2008). This model corresponds to the situation in which all taxa in the tree have evolved on a landscape with a single, constant optimum.

2B. Constant evolutionary bounds.-We simulated bounded evolution using a very similar approach to that of Revell (2007b) by imposing absolute bounds on the phenotype space. This was accomplished by using a selection model that can be represented as follows:

$$
w=\left\{\begin{array}{cc}
\text { if } y<l & 0.0 \\
\text { if } u>y>l & 1.0 \\
\text { if } y>u & 0.0
\end{array},\right.
$$

where $l$ and $u$ are the lower and upper limits of the bounded phenotype space, and $w$ and $y$ are as in scenario 2A.

The interaction between evolutionary rate and bounded space can be simulated either by varying the bounds or the evolutionary rate. We decided to fix the bounds at $u=10$ and $l=-10$, and vary the evolutionary rate by manipulating $\mu$ across simulations as for scenario 1 above. This model corresponds to the situation in which a range of phenotypes have roughly equivalent fitness, but any phenotypes outside the bounds have zero fitness, perhaps due to strong functional constraints.

3. Variable selection.-We also simulated three scenarios in which selection favored an optimal value, but in which that optimum was variable over the course of the simulation and among lineages.

3A. Fluctuating natural selection.-We simulated fluctuating natural selection with a model similar to scenario $2 \mathrm{~A}$, but in which the phenotypic optimum, $\theta$, moved according to a stochastic Brownian motion process with rates $\sigma_{\theta}^{2}=8.0 \times 10^{-5}, 8.0 \times 10^{-4}, 8.0 \times 10^{-3}$, and $8.0 \times$ $10^{-2}$. These values were arbitrarily chosen because they produce rates of drift of the optimum equal to the rates of drift of the phenotype expected in the pure drift simulations in scenario 1 above. Fluctuations occurred over time as well as independently among lineages. For all simulations we fixed the mutation rate at $\mu=0.002$ and the stabilizing selection parameter at $\omega^{2}=10$. This corresponds to the situation in which populations evolve to an optimal value, but the particular optimum wanders independently in each lineage.

3B. Stochastic peak shifts.-As in scenario 2A, we simulated evolving lineages under a stable fitness regime with a static optimum, $\theta$. However, we interrupted this conservative regime with occasional random shifts in the position of the optimum, $\theta$. For all simulations we fixed the probability of a shift of the optimum per generation at $\rho=0.0001$. The direction of optimum shift 
was random and drawn from a normal distribution with mean $=0.0$ and variance $\sigma_{\theta}^{2}=0.01,0.1,1.0$, and 10.0 (and also $\sigma_{\theta}^{2}=20.0$; see Results). We added the change in position of the optimum to the prior state for the optimum, which results in a correlation between prior and future states for the fitness peak in a given lineage. Peak shifts were both rare and in no way correlated with speciation events. As in scenario 2 above, we fixed the mutation rate at $\mu=0.002$ for these simulations. This corresponds to the situation where populations evolve towards a fitness optimum, which randomly (but rarely) changes to a new location. The rarity of change suggests that this model could also be called a niche conservatism model because stabilizing selection is conserved over time, and close relatives are expected to be found on the same or correlated fitness peaks (niches).

3C. Punctuated divergent selection.-For this model, we again evolved lineages under a stable fitness regime with a static optimum, $\theta$. However, at each bifurcation in the phylogenetic tree, we simulated strong divergent selection to a new optimum for one of the two daughter lineages. This was accomplished by shifting the optimum for the right daughter of each speciation event by drawing a new random optimum from a normal distribution with variance $\sigma_{\theta}^{2}=0.01,0.1,1.0$, and 10.0. (Because the trees were generated stochastically, it is irrelevant whether it is a random daughter node or invariably the rightmost daughter node that is subject to divergent selection to a new optimum.) Unlike scenario 3B, divergent selection occurred commonly and was invariably associated with a speciation event. Furthermore, the new selective environment is not expected to be correlated with the prior environment. As in scenarios $2 \mathrm{~A}, 3 \mathrm{~A}$, and $3 \mathrm{~B}$, above, we fixed the mutation rate at $\mu=0.002$ for these simulations. This model corresponds to a situation in which strong divergent selection usually accompanies each speciation event and thus could also be described as a peripatric speciation model, because speciation is accompanied by a new selective regime for one of the two resultant species and the new selective regime is uncorrelated with the prior regime. This might be the typical situation for peripherally isolated populations, such as island colonists. Our peripatric model is closely analogous to punctuational models developed for the analysis of comparative data in Oakley et al. (2005) and Lee et al. (2006).

Note that there is some terminological discordance between our model names and those of Hansen and Martins (1996), in which similar models are explored analytically. Principally, their "punctuated phenotypic change" corresponds to our "stochastic peak shifts" model. We prefer to reserve the term punctuation to refer to change associated with speciation events (sensu Gould and Eldredge, 1977; Gould 2002; Oakley et al., 2005). Furthermore, our "punctuated divergent selection" model differs from Hansen and Martins (1996) "change correlated with speciation events" model because in the latter, the prior and present states for the phenotypic optimum after speciation are correlated.
4. Time-dependent models.-Finally, we simulated evolution under conditions in which model parameters changed as a function of time.

4A. Heterogeneous rate genetic drift.-We simulated rate heterogeneity by simulating genetic $\mathrm{drift}$ (scenario 1 ) while varying the evolutionary rate by drift as a function of time. In these simulations, we set the mean mutation rate to $\bar{\mu}=0.002$. However, we varied the mutation rate as a linear function of time according to the equation:

$$
\mu_{t}=\bar{\mu} \cdot\left(1-b_{\mu} / 2+b_{\mu} \cdot t / t_{T o t}\right)
$$

in which $\mu_{t}$ is the mutation rate at time $t$, and $b_{\mu} / t_{T o t}$ is the rate of change in the mutation rate per generation ( $t_{T o t}$ being the total time of the simulation). We set $b_{\mu}=-2.0,-1.0,0.0$ (which is the same as scenario 1 above), 1.0, and 2.0. In general, $b_{\mu}<0.0$ represents a situation in which $\mu$ is initially high and decreases linearly over time, whereas $b_{\mu}>0.0$ represents a situation in which $\mu$ is initially low and increases over time. The rate of phenotypic evolution by genetic drift might be heterogeneous through time for any of a variety of biological reasons (such as higher solar radiation in tropical climes, concerted changes in the fidelity of DNA replication over time, trends in size or metabolic rate over time, and irreversible changes in ploidy or gene number; Rohde, 1978, 1992; Britten, 1986; Bromham et al., 1996; Revell and Harmon, 2008). Blomberg et al. (2003) also explored heterogeneity in the evolutionary rate with their ACDC (accelerate and decelerate) model for evolution by Brownian motion.

4B. Heterogeneous rate stochastic peak shifts.-We simulated heterogeneous stochastic peak shifts by repeating scenario $3 \mathrm{~B}$ while varying the probability of peak shifts as a function of time. In these simulations we set the mean probability of a peak shift in a given generation to $\bar{\rho}=0.0001$, as in scenario 3B. However, we varied the probability of shifting optimum as a linear function of time according to the equation:

$$
\rho_{t}=\bar{\rho} \cdot\left(1-b_{\rho} / 2+b_{\rho} \cdot t / t_{T o t}\right) .
$$

Terms are much as in scenario $4 \mathrm{~A}$ above. We set $b_{\rho}=$ $-2.0,-1.0,0.0$ (which is the same as scenario $3 \mathrm{~B}$ ), 1.0, and 2.0. We fixed the variance of niche shifts at $\sigma_{\theta}^{2}=$ 10.0 for all simulations. $b_{\rho}$ can be interpreted as is $b_{\mu}$, above. This model, in particular the case in which the probability of peak shifts is initially high but decreases over time $\left(b_{\rho}<0.0\right)$, is analogous to the niche occupancy scenario of Price (1997), in which niche divergence is high early in the adaptive differentiation of a group but slows over time as niches are filled.

\section{Calculation of Phylogenetic Signal}

For each simulation, we calculated phylogenetic signal using the $K$ statistic of Blomberg et al. (2003). We compared phylogenetic signal among simulation conditions 
for each evolutionary process, as well as among simulations of different evolutionary processes. Although, as noted above, we prefer Blomberg et al.'s (2003) K statistic, we also calculated Pagel's (1999) $\lambda$ and take note of the results as they are similar or different to those obtained for $K$.

\section{RESULTS}

Using Blomberg et al.'s (2003) K-statistic, phylogenetic signal varied widely across evolutionary processes (Figs. 2 to 5). However, differences among simulation conditions for a single process were often as large as the differences among processes (e.g., Fig. 3a, b; Fig. 4b; Fig. 5a, b). Phylogenetic signal was generally not significantly elevated over the neutral expectation of $K=1.0$ for any time-independent simulation conditions (Figs. 2 to 4). However, phylogenetic signal was elevated for some time-dependent simulation conditions (Fig. 5).

In particular, mean phylogenetic signal was not significantly different from $K=1.0$ under any conditions of constant-rate genetic drift (scenario 1; Fig. 2). For conditions of constant stabilizing selection (scenario 2A), phylogenetic signal was low when the strength of stabilizing selection was high ( $\omega^{2}$ was low; Fig. 3a) but increased under weaker stabilizing selection. For conditions of constant functional constraint (scenario 2B), bounded phenotype space did not decrease phylogenetic signal when the rate of evolution relative to the bounds of phenotype space was low (Fig. 3b), whereas phylogenetic signal was strongly decreased when the rate of evolution was higher. For conditions of fluctuating natural selection (3A), phylogenetic signal was somewhat low when the rate of fluctuation was low but increased with an increasing rate of Brownian motion fluctuation

\section{genetic drift}

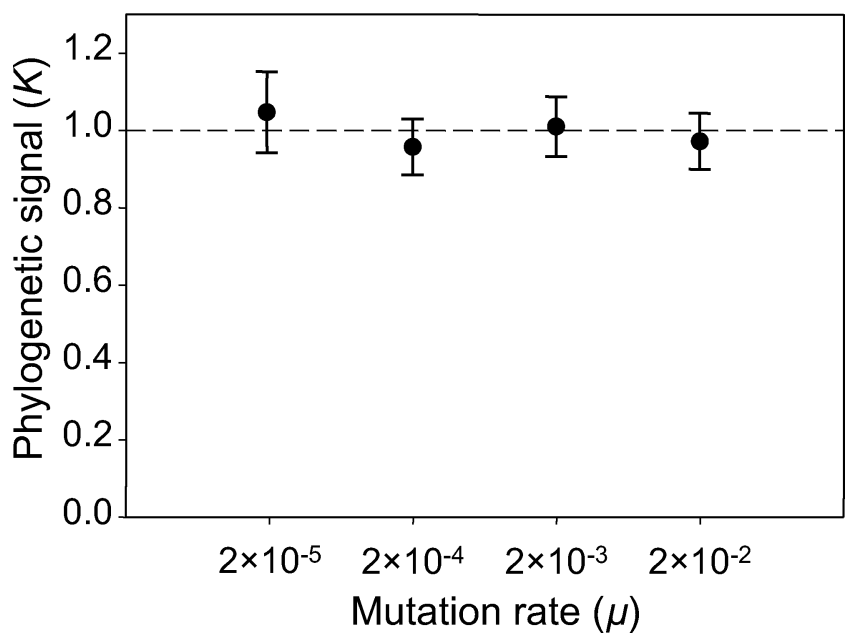

FIGURE 2. Mean phylogenetic signal, measured by Blomberg et al.'s (2003) $K$, for simulations of genetic drift using various values for the mutation rate, $\mu$. Error bars indicate $95 \%$ confidence limits for the mean value of $K$, and the horizontal dashed line is the expectation for $K$ under Brownian motion (1.0). Additional details are provided in the text.
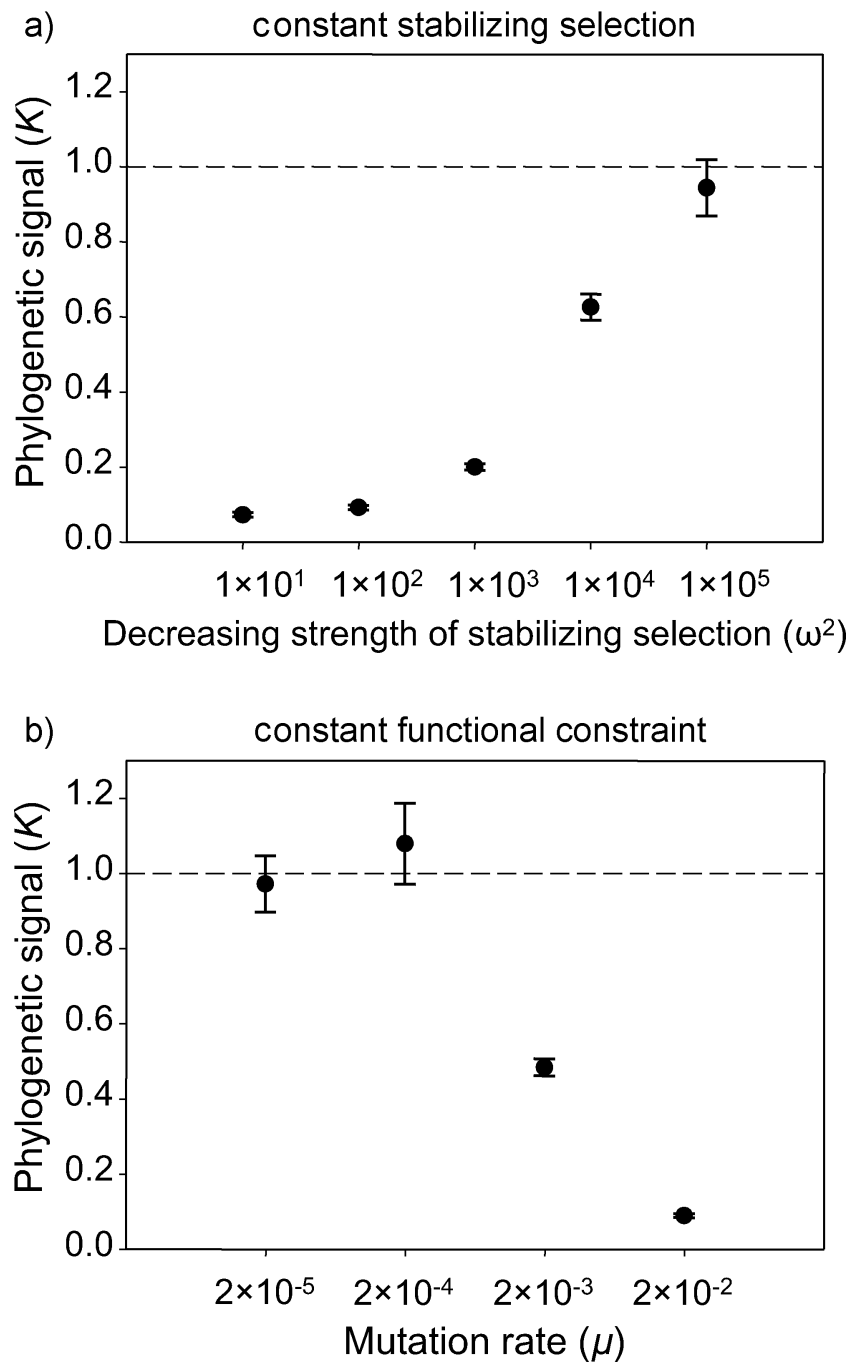

FIGURE 3. Mean phylogenetic signal for two constant selection processes, under different conditions. The processes are (a) constant stabilizing selection, in which the stabilized optimum is constant over time, for various values of the stabilizing selection parameter $\omega^{2}$. Larger values of $\omega^{2}$ indicate weaker stabilizing selection (the strength of selection increases with the inverse of $\omega^{2}$ ). (b) Constant functional constraint, simulated as bounded morphospace, for various mutation rates, $\mu$. Error bars and dashed lines are as in Figure 2. Additional details are provided in the text.

of the optimum (Fig. 4a). For conditions of stochastic peak shifts, phylogenetic signal was low when the size of peak shifts was small and high when the size of peak shifts was large (Fig. 4b). Because phylogenetic signal increased between $\sigma_{\theta}^{2}=1.0$ and $\sigma_{\theta}^{2}=10.0$ in this scenario, we decided to perform an additional simulation with the variance of peak shifts set to $\sigma_{\theta}^{2}=20.0$. We found that $\bar{K}$ did not significantly exceed 1.0 even as $\sigma_{\theta}^{2}$ increased (in fact, $\bar{K}$ was slightly lower for $\sigma_{\theta}^{2}=20.0$ than for $\sigma_{\theta}^{2}=$ 10.0). Finally, for conditions of punctuated divergent selection (scenario 3C), phylogenetic signal was low for all simulation conditions (Fig. 4c).

In contrast to the time-independent simulations, in the time-dependent simulations (scenario 4), phylogenetic 
a)

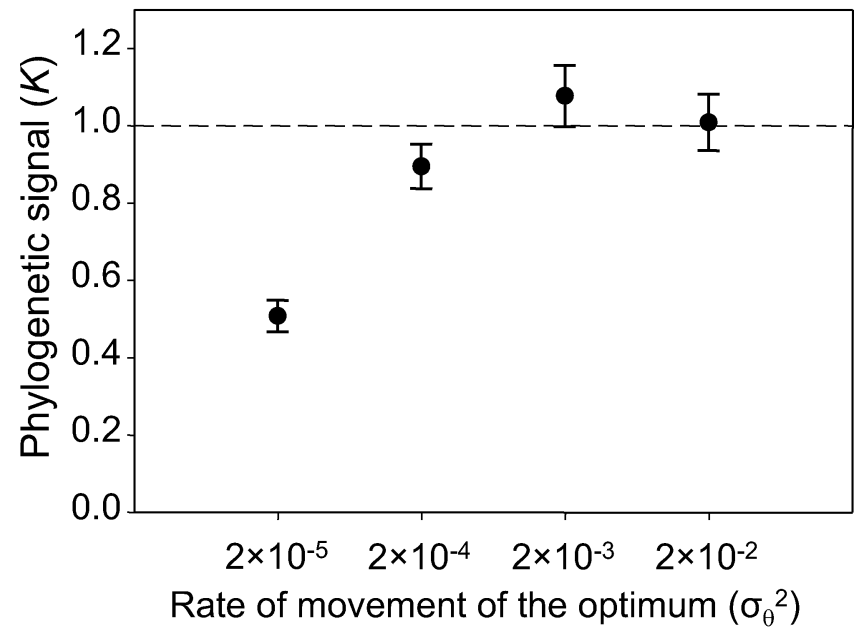

b)

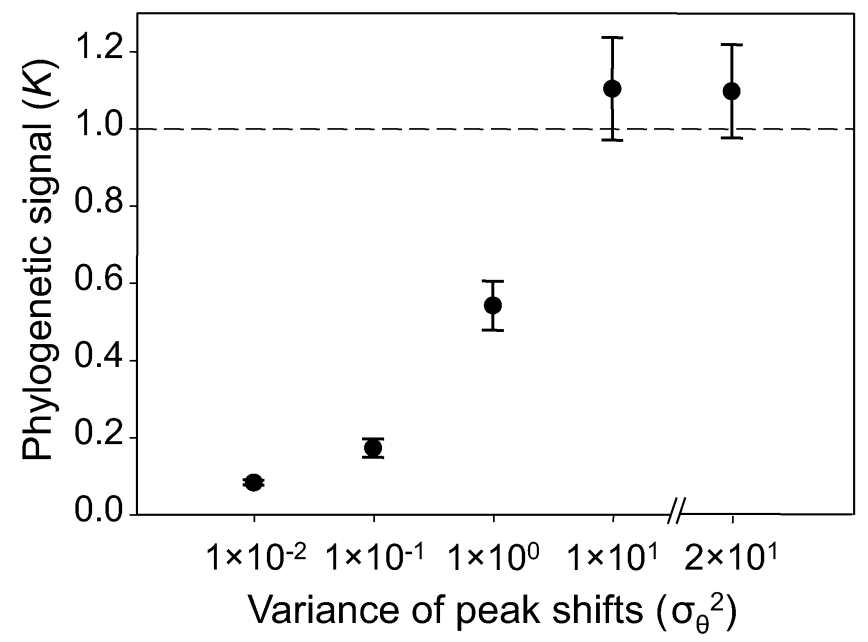

c) punctuated divergent selection

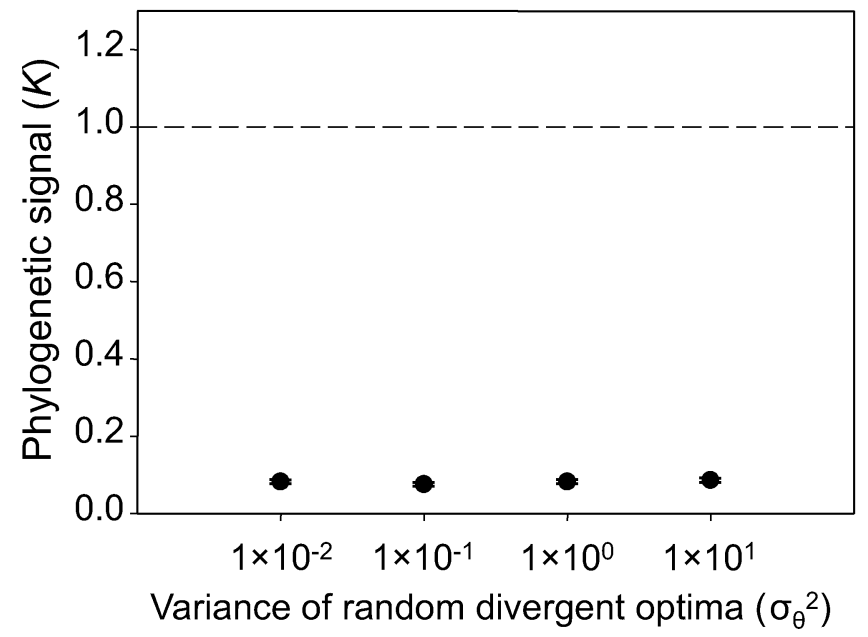

signal was significantly greater than 1.0 under some circumstances. In particular, heterogeneous rate genetic drift where the rate varied as a linear function of the time since the root (scenario 4A.) resulted in significantly elevated or depressed phylogenetic signal depending on the simulation conditions. Phylogenetic signal was low when the rate of drift was initially low but increased towards the present $\left(b_{\mu}>0.0\right)$ and high for a high initial rate that decreased towards the present $\left(b_{\mu}<0.0\right.$; Fig. 5a). Similarly, phylogenetic signal was low in the heterogeneous rate stochastic peak shift scenario (4B) when the rate of stochastic peak shifts (niche occupancy) was low and increased towards the present $\left(b_{\rho}>0.0\right)$, whereas phylogenetic signal was high when the rate of niche occupancy decreased over time $\left(b_{\rho}<0.0\right.$; Fig. $\left.5 b\right)$.

For all time-independent simulations (scenarios 1, 2, and 3), results obtained using Pagel's (1999) $\lambda$ (not shown) were quite concordant to those for $K$. However, in no evolutionary scenario was $\bar{\lambda}>1.0$, even as $K$ was severely elevated by time-dependent heterogeneity in the evolutionary process (Fig. 5a, b). Obviously, this leads to substantial discordance between the results for $K$ and $\lambda$ in the time-dependent simulations, due to the fact that $\lambda>1.0$ is usually not defined (Freckleton et al., 2002). Pagel (1999) suggested another parameter, $\delta$, which might be useful for heterogeneous rate situations such as those simulated in this study. However, $\delta$ does not provide a direct measure of phylogenetic signal. All subsequent discussion focuses on $K$ as the measure of phylogenetic signal.

\section{DISCUSSION}

Three main patterns were observed in the data. First, under the simplest scenario for the evolutionary process, constant-rate genetic drift, there was no relationship between the evolutionary rate and phylogenetic signal (Fig. 2). This suggests that low phylogenetic signal should not generally be interpreted as evidence of high evolutionary rate. Second, many different evolutionary processes produced similar phylogenetic signals (Figs. 2 to 5). This suggests that our ability to infer evolutionary process from the measurement of phylogenetic signal is probably limited. This is especially true for observations of low phylogenetic signal-as all but one evolutionary process simulated in this study produced depressed phylogenetic signal under some circumstances

FIGURE 4. Mean phylogenetic signal for three variable selection processes, under different conditions. The processes are (a) fluctuating selection, in which selection fluctuates over time and among lineages according to a Brownian process, for various rates of fluctuation $\left(\sigma_{\theta}^{2}\right)$. (b) Stochastic peak shifts, in which stable selection is occasionally disrupted by fitness peak shifts with various variances $\left(\sigma_{\theta}^{2}\right)$. (c) Punctuated divergent selection, in which each speciation event is accompanied by divergent selection of one of the two daughter species to a new optimum, drawn from distributions with various variances $\left(\sigma_{\theta}^{2}\right)$. Error bars and dashed lines are as in Figures 2 and 3. Additional details are provided in the text. 


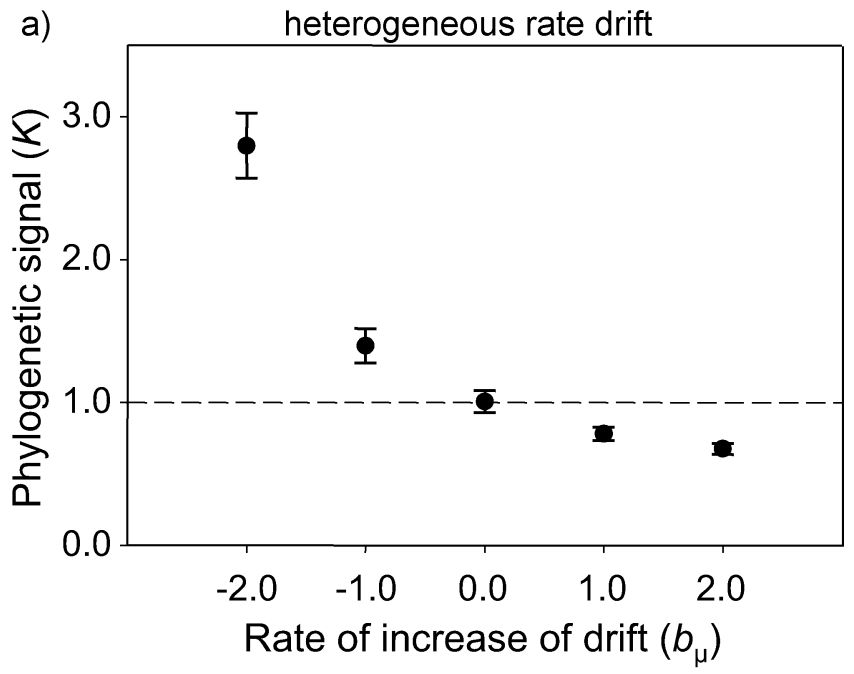

b) heterogeneous rate peak shifts

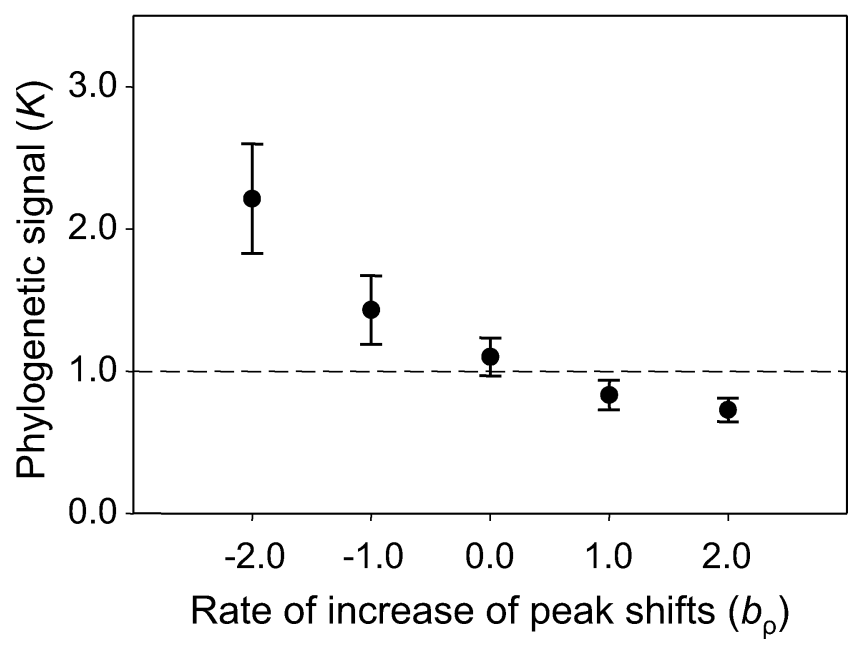

FIGURE 5. Mean phylogenetic signal for two time-dependent simulation models, under different conditions. The processes are (a) heterogeneous rate genetic drift, in which the rate of drift is initially high but decreases over time $\left(b_{\mu}<0.0\right)$, or vice versa $\left(b_{\mu}>0.0\right)$. (b) heterogeneous rate peak shifts, in which the probability of peak shifts is initially high in all lineages but decreases over time $\left(b_{\rho}<0.0\right)$, or vice versa $\left(b_{\rho}>0.0\right)$.

(Figs. 3 to 5). Finally, some processes increased phylogenetic signal relative to the neutral expectation. In particular, the scenario of a high rate of early peak shifts (niche occupancy) increased phylogenetic signal (Fig. 5b; $b_{\rho}<$ $0.0)$. Although fewer of the processes simulated in this study produced high phylogenetic signal, the prospect for evolutionary inference from high phylogenetic signal is limited by the fact that nonadaptive processes, such as heterogeneous rate genetic drift, can also produce similarly elevated signal (Fig. 5a; $b_{\mu}<0.0$ ).

\section{Phylogenetic Signal and Evolutionary Rate}

When evolution was unconstrained-in other words, under pure genetic drift-there was no relationship between the evolutionary rate and phylogenetic signal
(Fig. 2). This is not a surprising result as phylogenetic signal for continuous characters should be viewed as primarily a consequence of the evolutionary process, not the evolutionary rate (Blomberg and Garland, 2002; Blomberg et al., 2003). However, evolutionary rate can affect phylogenetic signal when evolution is bounded (Fig. 3b). It is well appreciated that evolutionary rate affects phylogenetic signal for discrete characters, such as genetic sequence data, when the number of states for the character is limited (Hillis and Huelsenbeck, 1992). This effect is more severe as the number of possible states for the character decreases or the rate increases (Donoghue and Ree, 2000; Ackerly and Nyffeler, 2004). Bounds on morphospace have an analogous effect (e.g., Whitehead and Crawford, 2006).

Under fluctuating selection when the position of the optimum moved by Brownian motion, phylogenetic signal was low when the rate of movement of the optimum was low (Fig. 4a). This is because when the rate of evolution is very low the optimum moves negligibly over the course of the simulation and as a consequence all the species at the tips are effectively experiencing stabilizing selection to the same optimum (as in scenario 2A; Fig. 3a). Variation among species means is then a consequence only of an inability to perfectly track the selective optimum (maladaptation), which will not have a phylogenetic component. This finding is consistent with Hansen and Martins' (1996) predictions regarding phylogenetic covariances under stabilizing selection to a static optimum (which is the limiting case for process $3 \mathrm{~A}$, as the rate of movement of the optimum approaches 0.0 ), and as well as with our findings for constant stabilizing selection (scenario $2 \mathrm{~A} ; \omega^{2}=10$ ).

In general, however, evolutionary rate did not affect phylogenetic signal for continuous characters under the assumption of most comparative methods-i.e., when the evolutionary process approximates Brownian motion (as in genetic drift, and some conditions of bounded evolution and fluctuating selection). However, under other circumstances, such as fluctuating natural selection when the rate of fluctuation is low and functional constraint when the bounds relative to the rate are small, evolutionary rate will affect phylogenetic signal.

\section{Phylogenetic Signal and Evolutionary Process}

Although rate can influence signal, a much larger source of variability in phylogenetic signal among our simulations arose from the evolutionary process. Phylogenetic signal was more or less invariant under only two processes. Phylogenetic signal was consistently high and not significantly different from $K=1.0$ for all conditions of constant-rate genetic drift (Fig. 2), whereas phylogenetic signal was consistently low for all conditions of divergent selection (Fig. 4c). Under all other scenarios, phylogenetic signal was low or high depending on the simulation conditions. No time-independent process seemed to increase phylogenetic signal over that expected under neutral conditions $(K=1.0)$. In contrast, heterogeneity of the evolutionary parameters over time 
both decreased and significantly increased the phylogenetic signal under different conditions (Fig. 5).

For constant stabilizing selection, phylogenetic signal was low (and, in fact, very near 0.0 ) for all conditions of strong stabilizing selection (Fig. 3a, low $\omega^{2}$ ). This finding is consistent with Hansen and Martins' (1996) finding that the phylogenetic covariances among species will be low for stabilizing selection to a single optimum. Phylogenetic signal increased under progressively weaker stabilizing selection around a constant optimum (larger $\omega^{2}$ ). As $\omega^{2}$ tends towards $\infty$, the simulation tends towards genetic drift (scenario 1) in which phylogenetic signal is invariably high (Figs. 2, 3). For evolution with fixed bounds, phylogenetic signal was low or high depending on the rate, as discussed above; however, the process of evolution with bounds generally tended to decrease signal, particularly as the evolutionary rate relative to the bounds was increased.

For fluctuating natural selection, in which the position of the optimum moved according to a Brownian motion process, phylogenetic signal was high so long as the rate of fluctuation was sufficiently high (Fig. 4a). When the rate of fluctuation was low, phylogenetic signal was decreased: these simulations reflect our findings for scenario $2 \mathrm{~A}$, discussed above.

We found low phylogenetic signal for simulations of rare stochastic peak shifts, when the size of rare niche shifts was small (Fig. 4b). This parallels the situation for fluctuating natural selection at a low rate (discussed above) and stasis of the fitness optimum (scenario 2A; Fig. 3a). As the size of rare niche shifts increased, so did phylogenetic signal (Fig. 4b). Punctuated divergent selection (divergent selection at speciation events) resulted in low phylogenetic signal under all conditions (Fig. 4c). Although we simulated divergent selection at speciation events, phylogenetic signal would also be low for any condition in which peak shifts are sufficiently common and random with respect to the prior state for the peak, as they are in our punctuated divergent selection simulations (scenario 3C).

Phylogenetic signal was generally decreased when the rate of evolution by genetic drift was initially low but increased over time $\left(b_{\mu}>0.0\right.$; Fig. $\left.5 a\right)$. This is because an increasing evolutionary rate tends to concentrate evolutionary change along branches towards the tips of the tree. This will tend to cause the variances of a character across the tips to increase without a concordant increase in the covariances among taxa. Conversely, high initial rate decreasing over time $\left(b_{\mu}<0.0\right)$ will increase the covariances among tips relative to that expected under constant-rate Brownian motion and will consequently increase phylogenetic signal (Fig. 5a).

Similarly, phylogenetic signal was decreased when the rate of fitness peak shifts, or niche shifts, was initially low but increased over time $\left(b_{\rho}>0.0\right.$; Fig. $\left.5 b\right)$. In this case, niche shifts are also concentrated towards the tips of the tree. The paucity of niche shifts on internal branches will decrease the covariances among tips relative to the neutral expectation and depress phylogenetic signal. Conversely, an initially high rate of niche differen- tiation (occupancy), which decreases towards the present $\left(b_{\rho}<0.0\right)$, will tend to increase phylogenetic signal relative to the neutral expectation. This is because most niche shifts, and thus most evolutionary changes, are concentrated towards the root of the tree. This will tend to increase the covariances among tips relative to the neutral expectation and thus enhance phylogenetic dependence (Fig. 5b). This latter niche occupancy model corresponds fairly closely to the niche differentiation model described in Price (1997), and perhaps expected during an adaptive radiation (Schluter, 2000).

\section{On the Evolutionary Parameters}

The specific values of the evolutionary parameters simulated in this study have not been justified. However, most are poorly known empirically (see Jones et al., 2003; Revell, 2007a). Even for situations in which estimates for the evolutionary parameters are available (such as for the mutation rate; Kimura, 1968), we could not practically use available rates because other parameters have been specified unrealistically, usually for computational reasons. For example, due to the small simulated effective population sizes in this study, realistically small mutation rates would lead to unrealistically negligible standing genetic variances (also discussed in Jones et al., 2003), and similarly negligible divergence among species. An implicit assumption of increasing one parameter while decreasing another is that so doing will have compensatory effect with regard to the evolutionary process and outcome. This assumption has been made in previous similar simulation studies (Jones et al., 2003, 2004; Revell, 2007a), and a compensatory effect has been demonstrated explicitly in one study (Revell, 2007a). However, future studies might consider using biologically realistic parameter values in similar analyses.

\section{Artifacts of Molecular Phylogeny Estimation and Phylogenetic Signal}

Blomberg et al. (2003) and Ives et al. (2007) point out two sources of bias in the empirical estimation of $K$. Error in phylogenetic topology and error in the estimation of species means will both, on average, downwardly bias the calculation of phylogenetic signal (Blomberg et al., 2003; Ives et al., 2007).

Consideration of the time-dependent simulations in this study, and in particular of the observation that factors affecting the covariances among species tend to affect $K$, suggests two other types of phylogenetic error that will also create bias in $K$ due to their tendency to cause misestimation of the time of shared history between taxa. These are model underparameterization and gene coalescence, and they work in the opposite direction of bias caused by topological error and error in the estimation of species means.

Sequence model underparameterization tends to cause early branches in a molecular phylogeny to be disproportionately shortened relative to later branches (Revell et al., 2005). This will cause estimated $K$ to be upwardly biased by decreasing the expected 
covariances among species relative to their expected values were the true tree known without error. Even if species exhibit only the expected amount of covariance under Brownian motion evolution on the true tree, these data will have covariance in excess of that predicted based on the (underestimated) lengths of internal branches, and will thus exhibit inflated phylogenetic signal.

In addition, gene lineage coalescence tends to precede speciation by, on average, $2 \cdot N_{e}$ generations, in which $N_{e}$ is the effective population size (Pamilo and Nei, 1988; Hein et al., 2005). This will not cause internal branches of a gene genealogy to be elongated compared to the branches of the true phylogeny, because all branches are, on average, lengthened by $2 \cdot N_{e}$ towards the root and shortened by $2 \cdot N_{e}$ towards the tips. However, tip branches are only lengthened by this phenomenon. This will tend to cause species expected variances (estimated from the gene genealogy) to be enlarged over their true values were the phylogeny (rather than the gene genealogy) known without error, and this occurs without an associated increase in species expected covariances. This will also cause an increase in the estimated value of $K$, because species expected covariances are underestimated relative to their true values in terms of species expected variances.

Thus, although topological error and error in the estimation of species means will tend to decrease estimated phylogenetic signal, we have identified two sources of upward bias in $K$ that are expected to result as artifacts of molecular phylogeny estimation. It should be established in a future study to what extent these four sources of error-topological error (Blomberg et al., 2003), error in the esimtation of species means (Ives et al., 2007), model underparameterization, and gene coalescenceare likely to affect the estimation of phylogenetic signal in empirical studies.

\section{CONCLUSIONS}

The simulation conditions of this study are a simplification of the real evolutionary processes that underlie the origin of phenotypic diversity among species. Nonetheless, simulations were performed over a broad range of conditions so as to, hopefully, accommodate a similarly broad range of possibilities for the evolution of natural populations. So doing suggests, at least preliminarily, that under the simplest conditions (neutral genetic drift) there is no relationship between evolutionary rate and phylogenetic signal-however, such a relationship can exist when evolution is not entirely neutral. Furthermore, different evolutionary processes can produce similar phylogenetic signal, and conversely similar evolutionary processes can produce very different evolutionary signatures in terms of phylogenetic signal.

Some authors might be tempted to circumvent this confusion by synonymizing high and low phylogenetic signal with conservatism and lability, respectively (e.g., Blomberg et al., 2003; Swenson and Enquist, 2007). The results of this study indicate that so doing should be strongly discouraged. This is because conservatism and lability imply an underlying consistency of process or rate. For example "lability" literally means "changeability," but phylogenetic signal was low for conditions of strong stabilizing selection to a single optimum as well as for conditions of strong, regular divergent selection; and phylogenetic signal was usually uncorrelated with rate. Similarly, "conservatism" literally means resistance to change, and suggests conservation of the niche or selective regime. However, little evidence was revealed in this study to support the general association of high signal with conservatism in the evolutionary process.

In fact, one of the most important generalizations of this study is that very different processes can produce similar results in terms of signal, and very different rates can produce similar phylogenetic signal. If one's goal is to assess the evolutionary process underlying the distribution of character states among species, a better approach might be to consider fitting alternative models for the evolutionary process to the data and tree (e.g., Hansen, 1997; Mooers et al., 1999; Harvey and Rambaut, 2000; Blomberg et al., 2003; Butler and King, 2004; Oakley et al., 2005; Lee et al., 2006; Estes and Arnold, 2007). Such models include punctuational divergence (Oakley et al. 2005; Lee et al., 2006), accelerating and decelerating evolutionary rate (Blomberg et al., 2003), and explicitly adaptive models for niche occupancy and evolution (Butler and King, 2004). Comparing alternative models for the evolutionary process should enable lability and conservatism to be defined explicitly in terms of the model parameters, estimated using likelihood, which themselves correspond to specific hypotheses for genetic drift, divergence, and stabilizing selection.

\section{ACKNOWLEDGMENTS}

Very useful comments on this article were provided by the Losos Lab at Harvard University: A. Harrison, T. Hsieh, B. Langerhans, J. Losos, L. Mahler, T. Ord, and Y. Stuart, as well as by T. Oakley, S. Blomberg, and D. Ackerly. These comments vastly improved the manuscript over earlier versions and were much appreciated.

\section{REFERENCES}

Abouheif, E. 1999. A method for testing the assumption of phylogenetic independence in comparative data. Evol. Ecol. Res. 1:895-909.

Ackerly, D. D., and R. Nyffeler. 2004. Evolutionary diversification of continuous traits: Phylogenetic tests and application to seed size in the California flora. Evol. Ecol. 18:249-272.

Blomberg, S. P., and T. Garland, Jr. 2002. Tempo and mode in evolution: Phylogenetic inertia, adaptation and comparative methods. J. Evol. Biol. 15:899-910.

Blomberg, S. P., T. Garland, Jr., and A. R. Ives. 2003. Testing for phylogenetic signal in comparative data: Behavioral traits are more labile. Evolution 57:717-745.

Bozinovic, F., A. P. Cruz-Neto, A. Cortés, G. B. Diaz, R. A. Ojeda, and S. M. Giannoni. 2007. Physiological diversity in tolerance to water deprivation among species of South American desert rodents. J. Arid. Environ. 70:427-442.

Britten, R. J. 1986. Rates of DNA sequence evolution differ between taxonomic groups. Science 231:1393-1398.

Bromham, L., A. Rambaut, and P. H. Harvey. 1996. Determinants of rate variation in mammalian DNA sequence evolution. J. Mol. Evol. 43:610-621.

Butler, M. A., and A. A. King. 2004. Phylogenetic comparative analysis: A modeling approach for adaptive evolution. Am. Nat. 164:683-695. 
Cavalli-Sforza, L. L., and A. W. F. Edwards. 1967. Phylogenetic analysis: Models and estimation procedures. Evolution 21:550-570.

Cheverud, J. M., M. M. Dow, and W. Leutenegger. 1985. The quantitative assessment of phylogenetic constraints in comparative analyses: Sexual dimorphism in body weight among primates. Evolution 39:1335-1351.

Crow, J. F., and M. Kimura. 1964. The theory of genetic loads. Pages 495-505 in Proceedings of the XI International Congress of Genetics (S. J. Geerts, ed.). Pergamon, Oxford, UK.

Donoghue, M. J., and R. H. Ree. 2000. Homoplasy and developmental constraint: A model and an example from plants. Am. Zool. 40:759769.

Estes, S., and S. J. Arnold. 2007. Resolving the paradox of stasis: Models with stabilizing selection explain evolutionary divergence on all timescales. Am. Nat. 169:227-244.

Falconer, D. S., and T. F. C. Mackay. 1996. Introduction to quantitative genetics. Prentice Hall, Essex, UK.

Felsenstein, J. 1985. Phylogenies and the comparative method. Am. Nat. 125:1-15.

Felsenstein, J. 1988. Phylogenies and quantitative characters. Annu. Rev. Ecol. Syst. 19:445-471.

Felsenstein, J. 2004. Inferring phylogenies. Sinauer, Sunderland, Massachusetts.

Freckleton, R. P., P. H. Harvey, and M. Pagel. 2002. Phylogenetic analysis and comparative data: A test and review of evidence. Am. Nat. 160:712-726.

Garland, T. Jr., P. H. Harvey, and A. R. Ives. 1992. Procedures for the analysis of comparative data using phylogenetically independent contrasts. Syst. Biol. 41:18-32.

Gittleman, J. L., C. G. Anderson, M. Kot, and H.-K. Luh. 1996. Comparative tests of evolutionary lability and rates using molecular phylogenies. Pages 289-307 in New uses for new phylogenies (P. H. Harvey, A. J. Leigh Brown, J. Maynard Smith, and S. Nee, eds.). Oxford University Press, Oxford, UK.

Gittleman, J. L., and M. Kot. 1990. Adaptation: Statistics and a null model for estimating phylogenetic effects. Syst. Zool. 39:227241.

Gould, S. J. 2002. The structure of evolutionary theory. Harvard University Press, Cambridge, Massachusetts.

Gould, S. J., and N. Eldredge. 1977. Punctuated equilibria: The tempo and mode of evolution reconsidered. Paleobiology 3:115-151.

Grafen, A. 1989. The phylogenetic regression. Philos. Trans. R. Soc. B 326:119-157.

Hansen, T. F. 1997. Stabilizing selection and the comparative analysis of adaptation. Evolution 51:1341-1351.

Hansen, T. F., and Martins, E. P. 1996. Translating between microevolutionary process and macroevolutionary patterns: The correlation structure of interspecific data. Evolution 50:1404-1417.

Hansen, T. F., and S. H. Orzack. 2005. Assessing current adaptation and phylogenetic inertia as explanations for trait evolution: The need for controlled comparisons. Evolution 59:2063-2072.

Harvey, P. H., and M. D. Pagel. 1991. The comparative method in evolutionary biology. Oxford University Press, Oxford, UK.

Harvey, P. H., and A. Rambaut. 2000. Comparative analyses for adaptive radiations. Phil. Trans. R. Soc. Lond. B 355:1599-1605.

Hein, J., M. H. Schierup, and C. Wiuf. 2005. Gene genealogies, variation and evolution: A primer in coalescent theory. Oxford University Press, Oxford, UK

Hillis, D. M., and J. P. Huelsenbeck. 1992. Signal, noise, and reliability in molecular phylogenetic analyses. J. Hered. 83:189-195.

Ives, A. R., P. E. Midford, and T. Garland, Jr. 2007. Within-species variation and measurement error in phylogenetic comparative methods. Syst. Biol. 56:252-270.

Jones, A. G., S. J. Arnold, and R. Bürger. 2003. Stability of the G-matrix in a population experiencing pleiotropic mutation, stabilizing selection, and genetic drift. Evolution 57:1747-1760.

Jones, A. G., S. J. Arnold, and R. Bürger. 2004. Evolution and stability of the G-matrix on a landscape with a moving optimum. Evolution 58:1639-1654.

Kimura, M. 1968. Evolutionary rate at the molecular level. Nature 217:624-626.

Lee, C., S. Blay, A. Ø. Mooers, A. Singh, and T. H. Oakley. 2006. CoMET: A Mesquite package for comparing models of continuous character evolution on phylogenies. Evol. Bioinform. Online 2:191-194.
Martins, E. P., and T. F. Hansen. 1997. Phylogenies and the comparative method: A general approach to incorporating phylogenetic information into the analysis of interspecific data. Am. Nat. 149:646-67.

Mooers, A. Ø., and D. Schluter. 1998. Fitting macroevolutionary models to phylogenies: An example using vertebrate body sizes. Contrib. Zool. 68:3-18.

Mooers, A. Ø., S. M. Vamosi, and D. Schluter. 1999. Using phylogenies to test macroevolutionary hypotheses of trait evolution in cranes (Gruinae). Am. Nat. 154:249-259.

Oakley, T. H., Z. Gu, E. Abouheif, N. H. Patel, and W.-H. Li. 2005. Comparative methods for the analysis of gene-expression evolution: An example using yeast functional genomic data. Mol. Biol. Evol. 22:40-50.

O'Meara, B. C., C. Ané, M. J. Sanderson, and P. C. Wainwright. 2006. Testing for different rates of continuous trait evolution using likelihood. Evolution 60:922-933.

Ossi, K., and J. M. Kamilar. 2006. Environmental and phylogenetic correlates of Eulemur behavior and ecology (Primates: Lemuridae). Behav. Ecol. Sociobiol. 60:53-64.

Pagel, M. 1999. Inferring the historical patterns of biological evolution. Nature 401:877-884.

Pamilo, P., and M. Nei. 1988. Relationships between gene trees and species trees. Mol. Biol. Evol. 5:568-583.

Price, T. 1997. Correlated evolution and independent contrasts. Phil. Trans. R. Soc. Lond. B 352:519-529.

Revell, L. J. 2007a. The G matrix under fluctuating correlational mutation and selection. Evolution 61:1857-1872.

Revell, L. J. 2007b. Testing the genetic constraint hypothesis in a phylogenetic context: A simulation study. Evolution 61:2720-2727.

Revell, L. J. 2008. On the analysis of evolutionary change along single branches in a phylogeny. Am. Nat. 172:140-147.

Revell, L. J., and L. J. Harmon. 2008. Testing quantitative genetic hypotheses about the evolutionary rate matrix for continuous characters. Evol. Ecol. Res. 10:311-331.

Revell, L. J., L. J. Harmon, R. E. Glor. 2005. Underparameterized model of sequence evolution leads to bias in the estimation of diversification rates from molecular phylogenies. Syst. Biol. 54:973-983.

Revell, L. J., M. A. Johnson, J. A. Schulte, II, J. J. Kolbe, and J. B. Losos. 2007. A phylogenetic test for adaptive convergence in rock-dwelling lizards. Evolution 61:2898-2912.

Rheindt, F.E., T. U. Grafe, E. Abouheif. 2004. Rapidly evolving traits and the comparative method: How important is testing for phylogenetic signal? Evol. Ecol. Res. 6:377-396.

Rohde, K. 1978. Latitudinal gradients in species diversity and their causes. I. A review of the hypotheses explaining the gradients. Biologisches Zentralblatt 97:393-403.

Rohde, K. 1992. Latitudinal gradients in species-diversity-The search for the primary cause. Oikos 65:514-527.

Rohlf, F. J. 2001. Comparative methods for the analysis of continuous variables: Geometric interpretations. Evolution 55:2143-2160.

Schluter, D. 2000. The ecology of adaptive radiation. Oxford University Press, Oxford, UK.

Silvertown, J., K. McConway, D. Gowing, M. Dodd, M. F. Fay, J. A. Joseph, and K. Dolphin. 2006. Absence of phylogenetic signal in the niche structure of meadow plant communities. Proc. R. Soc. B 273:3944.

Stearns, S. C. 1983. The influence of size and phylogeny on patterns of covariation among life-history traits in mammals. Oikos 41:173-187. Swenson, N. G., and B. J. Enquist. 2007. Ecological and evolutionary determinants of a key plant functional trait: Wood density and its community-wide variation across latitude and elevation. Am. J. Bot. 94:451-459.

Swenson, N. G., B. J. Enquist, J. Thompson, and J. K. Zimmerman. 2007. The influence of spatial and size scale on phylogenetic relatedness in tropical forest communities. Ecology 88:1770-1780.

Whitehead, A., and D. L. Crawford. 2006. Neutral and adaptive variation in gene expression. Proc. Natl. Acad. Sci. USA 103:5425-5430.

Zanne, A. E., C. A. Chapman, and K. Kitajima. 2005. Evolutionary and ecological correlates of early seedling morphology in East African trees and shrubs. Am. J. Bot. 92:972-978.

First submitted 6 November 2007; reviews returned 8 February 2008; final acceptance 28 April 2008

Associate Editor: Todd Oakley 